\title{
Alun THOMAS, Nomads and Soviet rule. Central Asia under Lenin and Stalin. Bloomsbury: Bloomsbury Academic, 2019, 257 p.
}

\author{
Xavier HALLEZ \\ Chercheur associé \\ EHESS, Paris (FR) \\ xavier.hallez@ehess.fr
}

Alun Thomas, actuellement enseignant d'histoire à l'université de Staffordshire (Royaume-Uni), étudie l'histoire moderne et contemporaine de la Russie et de l'Asie centrale. Le présent ouvrage est issu de sa thèse de doctorat soutenue en 2015, que l'auteur a complétée à partir des archives du Kirghizstan. Par la suite, il s'est notamment penché sur les questions de la mémoire et de la patrimonialisation et a publié un article intéressant à ce sujet : "An Empire Remembered? Collectivization and Colonialism in Mukhamet Shayakhmetov's "The Silent Steppe” ", in Dirk Göttsche (ed.), Memory an Postcolonial studies: Synergies and new directions across literatures from Europe, Africa and the Americas (Peter Lang, 2018).

Le travail d'Alun Thomas s'insère dans une réflexion déjà bien avancée sur les relations entre les populations nomades et le pouvoir bolchévique (Marco Buttino, La rivoluzione capovolta. L'Asia centrale tra il crollo dell'impero zarista e la formazione dell'URSS (Naples : L'ancora del mediterraneo, 2003) ; Isabelle Ohayon, La sédentarisation des Kazakhs dans l'URSS de Staline. Collectivisation et changement social (1928-1945) (Paris : Maisonneuve \& Larose-Institut Français d'Études sur l'Asie Centrale, 2006) ; Niccolo Pianciola, Stalinismo di frontiera. Colonizzazione agricola, sterminio dei nomadi e costruzione statale in Asia centrale (1905-1936) (Vicenza : Viella, 2009)).

Lobjet de sa recherche est clairement défini dans l'introduction :

The new Soviet state was confronted with the task of governing this nomadic population in Central Asia. This book recounts its efforts to do so. It divides these efforts across six themes or policy areas: common soviet perceptions of nomads and nomadism; land use and the distribution of land among nomads; the creation and enforcement of borders in nomadic regions; the confiscation and taxation of nomadic property; social policy among nomads and their inclusion into the framework of the state; and finally, the collectivisation of nomadic communities (p. 3).

Chacun des thèmes correspond à un chapitre spécifique.

Il est possible de formuler la question centrale d'Alun Thomas de la manière suivante : pourquoi le pastoralisme nomade n'a été ni traité comme un modèle économique cohérent, ni préservé en Asie centrale, alors qu'il représentait le mode de vie le plus répandu dans la région? Au travers des différents thèmes étudiés, il développe sa démonstration d'une unité de problématiques entre les populations nomades kazakhes, kirghizes et turkmènes, bien que la troisième soit plus rarement mentionnée. Les particularités nationales ne sont pas son sujet. 
Une introduction pose les bases de la démonstration de l'auteur afin de permettre aux lecteurs de comprendre la période soviétique en Asie centrale. Avec raison, Alun Thomas met l'accent sur la mobilité des nomades comme nœud des relations complexes entre ces populations et les administrations tsaristes, puis soviétiques. La construction de la perception des nomades par les autorités en a subi les conséquences. Cette présentation préliminaire, qui ne peut qu'être assez brève, n'est néanmoins pas tout à fait satisfaisante. Des termes centraux pour la période soviétique et en particulier celui de « baï » (riche) ne sont pas suffisamment contextualisés et historicisés. Le discours présentant les baï comme des exploiteurs, présent dans les années 1920, est apparu dès le XIX ${ }^{\mathrm{e}}$ siècle et montre des processus internes à la société kazakhe avant la période soviétique. Même si l'auteur a concentré son étude sur l'action des autorités russes, puis soviétiques, les nomades centrasiatiques apparaissent trop comme des sujets ou des victimes et non comme des acteurs des événements de la période 1917-1936. L’axe est celui de l'action d'un pouvoir exogène sur les populations nomades centrasiatiques. Cela induit l'auteur à présenter les différentes orientations politiques et les évolutions du pouvoir comme subies par ces populations et leurs implications dans celles-ci en sont minimisées.

Le premier chapitre est consacré aux perceptions du nomadisme par le pouvoir soviétique. Lauteur expose les tentatives des autorités pour catégoriser les nomades par touches successives : mode de vie, classe, économie, structures sociales et politiques. Malgré les enquêtes et les travaux réalisés, les nomades et le nomadisme restent mal connus et, en réalité, le pouvoir montre plutôt une forme d'indifférence à leur égard. La perception dominante est toujours celle d'une arriération et de l'incompatibilité de ce mode vie avec la modernité socialiste. Économiquement, «nomadism was also seen as highly unstable ». La conséquence en fut de nombreuses hésitations et changements dans les analyses : les défenseurs du nomadisme mettaient en avant l'adaptation à un environnement hostile impropre à l'agriculture et cette approche fut assez influente au début des années 1920, avant que la certitude d'un socialisme capable de modeler la nature selon ses besoins élimine cet argument. À partir de la deuxième moitié des années 1920, le débat se centre sur la manière d'œuvrer à une sédentarisation. Ainsi, la catégorie de nomade dans la construction de l'Asie centrale soviétique ne fut que très rarement utilisée, ce qui est bien montré dans les recensements.

Alun Thomas présente de nombreux éléments de ces débats, mais il parle peu de la perception générale de l'élevage par rapport à l'agriculture, qui fut toujours particulière en Russie (le cas bouriate est en ce sens révélateur), ou des conflits au Turkestan au sujet de la priorité accordée à la culture du coton face à l'élevage.

Le deuxième chapitre est consacré à l'espace nomade et plus spécifiquement à la question agraire : l'auteur offre un large panorama des différents niveaux impliqués. Les autorités soviétiques reprirent la conclusion de leurs prédécesseurs sur un espace immense et peu exploité par les nomades. Toutefois, la politique affichée de décolonisation imposait une autre analyse, à savoir une dépossession des bonnes terres par les colons et les difficultés en conséquence des nomades à pratiquer l'agriculture. Mais les mesures de décolonisation mises en œuvre et décrites par l'auteur sont symptomatiques del'ambiguïté du pouvoir. Les réformes agraires aboutissent finalement à favoriser l'agriculture et les grands projets étatiques. Malgré des institutions qui prêtaient attention au cas particulier 
des nomades (notamment le Comité fédéral à la terre), le discours dominant devint celui d'un nomadisme préservé et promu par les « exploiteurs » pour continuer à dominer le peuple. La régulation de l'usage des terres et de l'eau devint un slogan de libération de la politique agricole soviétique et la sédentarisation devenait une manifestation de la guerre de classe.

Le troisième chapitre traite des mobilités qui ont toujours posé problème au pouvoir colonial ou soviétique. Alun Thomas insiste sur le rapport particulier des nomades à la frontière - dû à leur mobilité saisonnière - et sur la volonté des autorités de réduire ces déplacements. Plusieurs cas de conflits territoriaux autour dela délimitation des frontières (russo-kazakh et turkmèno-kazakh) sont clairement exposés : les frontières instaurées ne correspondent pas aux besoins et aux habitudes des nomades et la délimitation est rendue compliquée du fait du mélange des populations. L'option d'abandonner la délimitation d'une frontière ou de la rendre poreuse ne fut pas envisagée. Au contraire, le national s'impose et il n'est pas question du nomadisme : le contrôle du territoire devient l'essentiel. La politique nationale bolchévique repose sur l'attribution d'une république à chaque nationalité reconnue :

But within these national territories political considerations often gave way to economic ones. The educated, urban Central Asians working for the party could empathise with many nomads of their own nation only a little more than could members of other nationalities. Once given their own juridisctions, these administrators were incentivised to emphasise the importance of borders that demarcated and protected their authority but complicated nomadic life (p. 103).

Le chapitre quatre aborde la question de l'imposition des nomades. Dans un premier temps, la politique de décolonisation aboutit à une taxation réduite pour les nomades. Mais très vite, les choix furent l'objet de négociations entre différentes institutions avec des objectifs politiques variables. Progressivement l'orientation de la politique fiscale fut déterminée de manière à forcer les changements sociaux et pousser à la sédentarisation :

As in all policy areas, the Party lacked the infrastructure and information necessary to unduce transformation among nomads in anything but the most blunt and disruptive way (p. 134).

Le chapitre cinq montre plusieurs initiatives du pouvoir soviétique pour mobiliser les nomades dans leur projet politique. Lauteur utilise la sentence suivante :

In order to mobilise, the state went mobile » (p. 142). Au travers des expériences de la Caravane rouge en 1922 et des yourtes rouges plus pérennes, il estime que « one of the intended effects of the decision to go mobile, part of an effort to mobilise nomads for socialist construction, was that the nomads themselves would demobilise and settle ; this is shown by the red yurts' advocacy for agriculture as well as the wider connection made between cultural progress and settlement (p. 155).

Il ajoute que :

[...] one of the advantages of this "mobilisational" interpretation is that it denotes a deepening relationship between the behaviour of the population and the objectives and functions of the state, a strengthening of the state (p. 140). 
Le sixième et dernier chapitre marque la fin du nomadisme en Asie centrale soviétique. Le choix fut celui d'une modernisation imposée par la collectivisation et la sédentarisation forcées, alors qu'auparavant la sédentarisation était censée être promue par des politiques collatérales (propriété de la terre, imposition, éducation...).

Alun Thomas conclut sur l'idée d'une modernisation créant la nationalité sur des éléments comme la langue, mais pas sur le mode de vie nomade. La nouvelle géographie de l'Asie centrale bouscula l'espace antérieur des communautés nomades. La volonté de l'auteur d'analyser la soviétisation de l'Asie centrale au travers de la catégorie du nomadisme apporte un regard enrichissant sur de nombreuses questions et permet d’approcher la diversité des discours dans les années 1920. 